\title{
Optimización de Redes Eléctricas Mediante la Aplicación de Algoritmos Genéticos
}

\author{
Daniel O. Anaut, Guillermo F. di Mauro, Gustavo Meschino y Juan A. Suárez \\ Universidad Nacional de Mar del Plata, Facultad de Ingeniería, Departamento de Ingeniería \\ Eléctrica, Juan B. Justo 4302, Mar del Plata, Pcia. Buenos Aires-Argentina \\ (e-mail: danaut@fi.mdp.edu.ar)
}

\begin{abstract}
Resumen
Este trabajo plantea la optimización de la configuración topológica de redes eléctricas de distribución secundarias tendiente a minimizar las pérdidas técnicas por efecto Joule, utilizando Algoritmos Genéticos. Mediante la aplicación sobre dos sistemas de distribución, se encontró que el método de optimización utilizado es capaz de hallar la solución óptima entre todas las posibles combinaciones que ofrecen las maniobras de los interruptores, comprobándose su flexibilidad para adaptarse a las restricciones de radialidad y nivel de tensión, involucrando un tiempo menor que el necesario para una búsqueda exhaustiva. Durante el desarrollo de la aplicación se validaron los operadores genéticos, determinándose cuáles eran aquéllos que proporcionaron el mejor desempeño en la búsqueda de la solución. Los resultados indican la factibilidad y viabilidad de la aplicación en la configuración óptima de sistemas de distribución eléctrica.
\end{abstract}

Palabras clave: algoritmos genéticos, optimización, redes de distribución eléctrica, flujo de carga

\section{Optimization of Electrical Networks using Genetic Algorithms}

\begin{abstract}
This work proposes the optimization of the topological configuration of secondary electrical distribution networks to minimize technical losses produced during their exploitation due to Joule effect, using for this Genetic Algorithms. Through the application of the method to two systems, it was found that the optimization technique is able to find the optimum solution among all the possible combinations that switch operations offer. Its flexibility to adapt to the restrictions of radiality and voltage level in less time than that of exhaustive search is also proved. During the development of the application the genetic operators were validated, determining those that gave the best performance in the search of the solution. The results show the feasibility and viability of the application for the optimum configuration of electrical distribution systems.
\end{abstract}

Keywords: genetic algorithms, optimization, electrical distribution networks, load flow 


\section{INTRODUCCIÓN}

Dentro de los aspectos a tener en cuenta en la explotación de una red de distribución de energía eléctrica, la reducción de las pérdidas por efecto Joule (pérdidas técnicas) resulta ser de vital importancia para mejorar la eficiencia en la prestación del servicio. Las redes de distribución eléctrica están configuradas, en su mayoría, en forma radial para una fácil operación y conveniente coordinación de las protecciones.

Bajo condiciones normales de explotación, la topología de la red puede ser modificada con el objeto de reducir las pérdidas, mejorar el perfil de tensión del alimentador y aumentar su confiabilidad, mientras se satisfagan todos los requerimientos de carga y se mantenga la operación del sistema en forma radial. Estos requerimientos conducen a un complejo problema de optimización que, planteados en términos matemáticos, resulta entero no-lineal. La solución óptima exacta puede ser obtenida al examinar todas las posibles combinaciones de apertura o cierre de interruptores de un $\mathrm{SD}$, demandando su resolución un excesivo tiempo de operación computacional.

Los principales métodos de resolución presentados en la literatura (Hong y HO, 2003) son de tres tipos: optimización, heurísticos y estadísticos. La mayoría de los métodos propuestos convergen a una solución óptima local, por lo cual la convergencia al óptimo global no es garantizada.

Como objetivo para este trabajo se plantea resolver el problema de optimización del SD para mínimas pérdidas a través de AG, teniendo en cuenta restricciones que hacen a la explotación real de una red como lo es la caída de tensión máxima, la radialidad del sistema y en un tiempo computacional aceptable.

Mediante un análisis cuantitativo, la cuestión a resolver, se formula como un problema de optimización entera con una función objetivo cuadrática y ecuaciones lineales de compromiso con fórmulas estado-dependientes. Las restricciones de radialidad en la operación del sistema, aislamiento, potencia de la fuente, tensión y corriente se formulan teniendo en cuenta lo planteado en Radha et al. (2003).

La técnica empleada consiste en analizar cada malla de un SD en su conformación inicial representando el estado de los interruptores, lo cual constituye un conjunto de configuraciones iniciales (población inicial del AG). En cada iteración y a partir de la población corriente, usando los operadores de selección, recombinación y mutación, se obtiene un nuevo conjunto de configuraciones (nueva población). En cada una de ellas se encuentran configuraciones de mejor calidad pretendiéndose hallar una solución (configuración) óptima global.

En los AG, la información sobre el objetivo a alcanzar es representada mediante la función de evaluación que se procurará minimizar. En este caso se propone una función que no sea sobreaproximada, la cual conduciría directamente a una solución irrealizable.

Para la implementación del método se desarrolló un algoritmo utilizando una plataforma de software de base matemática. La técnica propuesta fue probada en dos SD reportados en la literatura, para poder ser comparados en la obtención de la solución optima.

A los efectos de optimizar el rendimiento del AG, sus parámetros deben ser elegidos adecuadamente para cada problema. Así, una exploración exhaustiva se ha realizado justificando los parámetros finales de simulación: tipo de selección, cruzamiento, tasa de reproducción y mutación.

\section{Algoritmos genéticos}

El proceso de modelar la red en términos interpretables por los AG consiste en codificar a los individuos. Estos se representan como cadenas que indican qué interruptores deben ser abiertos para obtener el valor de pérdidas correspondiente (función de aptitud). Por ejemplo, si el SD esta 
compuesto por cinco mallas y los interruptores a abrir por cada una de ellas son los números 8,11 , 16,25 , y 31 , la cadena (denominada cromosoma) que representa la configuración resultante de la apertura de estos interruptores es la siguiente:

Cromosoma resultante con codificación binaria

\begin{tabular}{|l|l|l|l|l|l|l|l|l|l|l|l|l|l|l|l|l|l|l|l|l|l|l|l|l|}
\hline 0 & 1 & 0 & 0 & 0 & 0 & 1 & 0 & 1 & 1 & 1 & 0 & 0 & 0 & 0 & 1 & 1 & 0 & 0 & 1 & 1 & 1 & 1 & 1 & 1 \\
\hline Interruptor 8 & \multicolumn{10}{|l|}{ Interruptor 11} & \multicolumn{1}{|l|}{ Interruptor 16} & \multicolumn{1}{|c|}{ Interruptor 25} & Interruptor 31 \\
\hline
\end{tabular}

Cromosoma resultante con codificación entera

$$
\begin{array}{|l|l|l|l|l|}
\hline 8 & 11 & 16 & 25 & 31 \\
\hline
\end{array}
$$

Como el AG trabaja con un conjunto (población) de cadenas (posibles soluciones) es decir, múltiples puntos de búsqueda, constituye un método de búsqueda paralelo. Después de la convergencia, las cadenas son decodificadas en las variables originales y las soluciones son obtenidas.

Para evaluar a los individuos se requiere de una función de aptitud que determinará qué tan buena es una solución para resolver el problema. La función para este trabajo está compuesta por dos partes. La primera, por un cálculo que reconstruye el SD a partir de los interruptores a abrir de un individuo definido en cada una de las cadenas a evaluar. La segunda, por un cálculo de flujo de potencia, el cual se encargará de determinar las pérdidas de potencia para la configuración resultante del SD que surja de abrir los interruptores indicados por la solución propuesta.

De este modo, un individuo estará representado por un cromosoma compuesto por genes que codifican los interruptores que deben ser abiertos en el SD. La aptitud de este individuo estará representada por el valor de pérdidas de potencia que esta cadena, con su combinación de interruptores a abrir, genera en el SD. Por ende, cuanto menor sea el valor de pérdidas, mayor será la aptitud del individuo.

Las mejores soluciones (individuos) serán seleccionadas y cruzadas aplicando los operadores genéticos correspondientes. De esta manera se produce una nueva población de posibles soluciones, la cual reemplazará a la anterior, verificando la propiedad de que la población siguiente posea mejores características en comparación con la población anterior. Así, a lo largo de las generaciones, las buenas características se propagan a través de la población

\section{Antecedentes}

La adopción del cálculo del flujo de potencia puede ser formulada como un modelo de programación matemático. El llamado algoritmo de permutación de rama parece ser el método de solución aproximado más eficiente, mientras que otras mejoras han sido propuestos por Goswami y Basu (1992) y Chiang y Jumeau (1990a, 1990b).

Si bien algunos algoritmos propuestos pueden resolver el problema con bastante menos tiempo computacional, los resultados del cálculo en la mayoría de ellos son sólo aproximaciones a óptimos locales. Por tanto, con la finalidad de encontrar un óptimo global aproximado, se ha aplicado el método de "recocido (templado) simulado" propuestos por Kashem et al. (1999).

Sin embargo, el tiempo computacional es demasiado grande para conseguir una solución satisfactoria. Otros autores (Taylor y Lubkeman, 1990), con la finalidad de reducir el tiempo computacional, aplicaron un SD experto usando reglas heurísticas, disminuyendo así el espacio de búsqueda.

El algoritmo llamado "técnica de medición de distancia" propuesto por Kashem et al. (2000), permitió encontrar un primer lazo y así determinar en él, una opción de cierre para mejorar el balance de 
carga, permitiéndole alcanzar una configuración cercana a la óptima.

La reconfiguración de la red también fue formulada como programación multiobjetivo por Hsiao y Chen (2001), al considerar las pérdidas de potencia, la seguridad del SD y la calidad de potencia. Los desempeños del SD son expresados en conjuntos difusos para representar su naturaleza imprecisa. Una programación evolutiva fue entonces introducida para determinar la solución óptima.

El algoritmo de "recocido simulado" ha sido incorporado con "búsqueda Tabú" (Jeon y Kim, 2000) para la reducción de pérdidas. La "búsqueda Tabú" intentó determinar una solución mejor en el modo de un algoritmo descendente, pero no hubo garantía para la propiedad de convergencia.

Lin et al. (2000) han presentado un AG redefinido, en el cual el cruzamiento convencional y los esquemas de mutación fueron redefinidos por un mecanismo de competición para minimizar pérdidas.

En Morton y Mareels (2000), se presenta el método de "fuerza bruta", el cual se basa en la teoría de grafos y una matriz de sensitividad de corrientes. Este método garantizó una solución óptima global pero a costa de una búsqueda exhaustiva.

Hong y HO (2003), proponen un nuevo método basado en AG para la reconfiguración de la red de distribución. Los métodos tradicionales usaron los estados de los interruptores para codificar y decodificar los genes (bits o dígitos) de un cromosoma, lo cual requirió una "comprobación de malla extra" para identificar la existencia de lazos (mallas) en las soluciones temporarias. En este trabajo, se agrega un nodo de codificación-decodificación basado en el número Prufer, de modo de obtener una estructura radial sin una "comprobación de malla extra" en el SD.

Hong y Ho (2005), proponen una herramienta para la reconfiguración de distribución basado en la integración de los métodos de programación multiobjetivo fuzzy y algoritmos genéticos. El problema es planteado como una programación "entera mixta".

Finalmente, en Enacheanu et al. (2008), se utilizan los conceptos teóricos de los gráficos de Matroid para mejorar la eficiencia de los operadores de mutación y crossover.

\section{METODOLOGÍA}

Para evaluar el desempeño de los AG, se probaron dos SD con diferentes topologías: una red de distribución de 12,66 kV con 33 barras (Sistema 33-B), propuesta en Baran y Wu (1989) y un tramo del SD de la Compañía Pacífica de Gas y Electricidad de San Francisco, Estados Unidos de 12,66 kV con 69 barras (Sistema 69-B), propuesta en Chiang y Jumeau (1990a). La primera red consiste de un nodo fuente, 31 nodos o barras de carga, 31 secciones de líneas alimentadoras y 5 líneas vinculantes (Figura 1).

El segundo SD consta de un nodo fuente, 68 nodos de carga, 68 secciones de líneas alimentadoras, y también 5 líneas vinculantes.

En la red, cada sección de línea es asociada con un interruptor. Sobre cada uno de estos SD se aplicó el método de optimización mediante AG, para reconfigurar la red, con mínimas pérdidas, mantenimiento las siguientes condiciones:

1) La configuración de la red debe ser radial para que las protecciones operen adecuadamente.

2) Todos los tramos de los alimentadores deben estar energizados y con sus transformadores de distribución conectados.

3) No debe haber sobrecarga en ninguna parte de la instalación. 


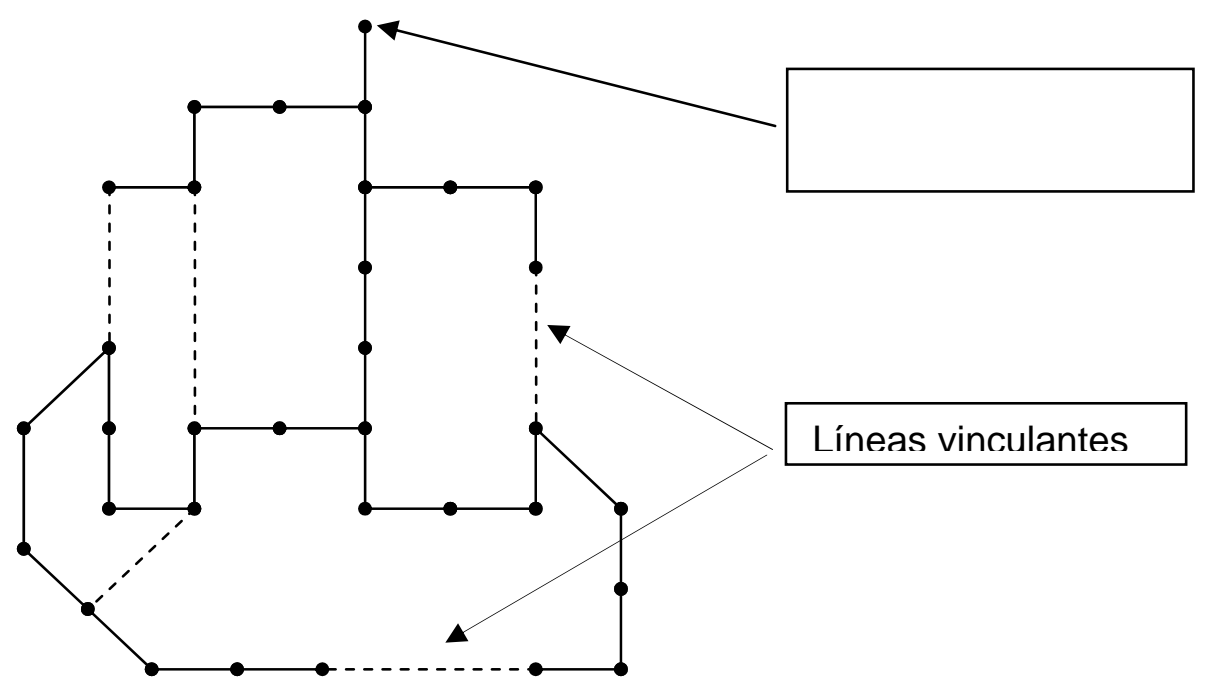

Fig. 1: Red de distribución de 12.66kV con 33 barras (Sistema 33-B)

\section{Flujo de Potencia}

Los métodos tradicionales para resolver flujos de potencia estudian el problema de reconfiguración de redes aplicando cálculos simplificados (Baldwin y Lewis, 2004). Dichas aproximaciones desprecian términos que si bien no afectan en demasía los resultados finales con respecto a los cálculos que emplean fórmulas completas, cobran importancia en el caso de SD de grandes dimensiones. Por ello, el tiempo computacional involucrado al aplicar un método exacto es excesivo para su utilización en tiempo real.

En este trabajo se utiliza un cálculo de flujo de potencia más exacto ya que, a diferencia de las técnicas previamente utilizadas, la implementación de los AG radica fundamentalmente en la exactitud del cálculo del Flujo de Potencia (FdP) para hallar las pérdidas de potencia. Dicho FdP se encarga de evaluar las configuraciones (cadenas) que tienen la potencialidad de resolver el problema y asociarle un valor de pérdidas de potencia (aptitud), que acompaña a cada solución candidata. Por lo tanto, este valor debe ser lo más exacto posible, para diferenciar de modo preciso las distintas cadenas que componen la población en el AG y ser explotados al máximo los beneficios de su aplicación.

A partir de los antecedentes estudiados, se analizaron las distintas metodologías para el cálculo del flujo de potencia y se encontró que las más apropiadas eran las aplicadas por Baran y Wu (1989), ya que tienen en cuenta todas las potencias involucradas en cada línea, en las cargas y sus correspondientes pérdidas de potencia.

Las ecuaciones de flujo de potencia adoptadas son:

$$
\begin{aligned}
& P_{i+1}=P_{i}-r_{i} \frac{P_{i}^{2}+Q_{i}{ }^{2}}{V_{i}^{2}}-P_{l i+1} \\
& Q_{i+1}=Q_{i}-x_{i} \frac{P_{i}^{2}+Q_{i}^{2}}{V_{i}^{2}}-Q_{l i+1} \\
& V_{i+1}^{2}=V_{i}^{2}-2\left(r_{i} P_{i}+x_{i} Q_{i}\right)+\left(r_{i}^{2}+x_{i}^{2}\right) \frac{P_{i}^{2}+Q_{i}^{2}}{V_{i}^{2}}
\end{aligned}
$$


El procedimiento para el cálculo es:

1- Se fijan los valores de tensión en todos los nodos solamente para la primera corrida en 1 pu.

2- Se calculan las potencias de todas las ramas que componen el SD.

3- Se actualizan los valores de tensión en todos los nodos.

4- Se vuelve al punto 2 hasta que el criterio de tolerancia es alcanzado.

Una vez alcanzado este último punto se calculan las pérdidas del SD, sumando las pérdidas de potencia que se producen en cada rama, de acuerdo a la expresión:

Pérdidas totales $=\sum_{\mathrm{i}=0}^{\mathrm{n}-1} \mathrm{R}_{\mathrm{i}, \mathrm{i}+\mathrm{1}} \frac{\left(\mathrm{P}_{\mathrm{i}}^{2}+\mathrm{Q}_{\mathrm{i}}^{2}\right)}{\left|\mathrm{V}_{\mathrm{i}}\right|^{2}}$

Finalizado este proceso la salida entregará el valor de la aptitud (pérdidas) del individuo bajo evaluación. Este valor que es único para cada configuración, y lo identifica de los demás, es una medida que indica qué tan buena es esta configuración para optimizar el problema.

\section{Modelado y codificación del SD}

Cada SD se modela con tramos de conductores (ramas o líneas) y carga de potencia constante en los nodos o barras. Cada rama a su vez se modela con su impedancia de secuencia positiva, mientras que las cargas se las modela con las demandas activa y reactiva constantes. El sistema trifásico se supone balanceado, por lo que se modela por fase y la red debe poder ser configurada radialmente.

Una alternativa de codificación de un SD en forma de árboles se ofrece en Hong y HO (2003), con el Número Prüfer. Este método genera soluciones que no son factibles de cumplir como la restricción de radialidad, por lo que es necesario desarrollar un algoritmo extra que compruebe que cada solución aportada cumpla la restricción anteriormente mencionada. Otras falencias de esta codificación es la poca similitud entre las aristas de dos árboles con codificaciones Prüfer similares y la dificultad de adaptar el método cuando el grafo de origen no es el completo. Además, cuando se aplican los operadores genéticos de cruzamiento y mutación a dos cadenas que ya verifican radialidad, la descendencia de éstas no necesariamente la verifica, por lo que nuevamente es necesario aplicar ese algoritmo extra de verificación a la descendencia producto del cruzamiento y/o mutación. En otras palabras, el algoritmo extra de verificación de radialidad es aplicado tanto a las soluciones "padres" como a las soluciones "hijas", por lo que el tiempo computacional se incrementa proporcionalmente al número de individuos componentes de cada población o generación.

Poniendo en práctica este método para los SD bajo estudio, el algoritmo extra de verificación de radialidad puede expresarse en forma de una matriz equivalente que contemple todas las combinaciones posibles de conectividad entre los nodos que componen el SD bajo estudio. Dicha matriz es introducida en la función de evaluación, la cual es responsable de retornar el valor de pérdidas de potencia correspondiente a una cadena determinada. Esta matriz ahorra de manera efectiva la elaboración de ese algoritmo ya que al aplicarla una sola vez, garantiza que cualquier combinación de interruptores a abrir que ingresa a la función de evaluación, responde a un SD radial. Para la confección de dicho algoritmo se siguió los lineamientos aportados por Goswami y Basu (1992) y Li et al. (2002).

Con respecto a la topología de las redes y al procedimiento de definición de las mallas que se generan en cada SD, la metodología más acertada en lo que se refiere a simplicidad, es aportada por Ah King et al. (2003). Este es el proceso que con algunas modificaciones se aplicó en ese trabajo. Así, para el sistema 33-B surgen 5 interruptores abiertos. Si se cierran esos interruptores se 
forman 5 lazos o mallas, cada una compuesta por ramas que se numeran partiendo desde el nodo origen o fuente. El nodo que recibe el flujo de corriente indicará el número de la rama, de forma que siempre queda contemplado que la configuración original sea una opción. Las mallas así definidas consideran la apertura de un interruptor y sólo uno por malla.

De este modo queda conformada una cadena donde cada elemento representa una posición en la expresión de la malla correspondiente, la cual indica el número del interruptor a abrir. Así, cualquier combinación que arroje responde a una configuración radial.

\section{CADENA: $\{$ [Malla 1] [Malla 2] [Malla 3] [Malla 4] [Malla 5] $\}$}

Por ejemplo, si el AG arroja como resultado la cadena $\left\{\begin{array}{lllll}3 & 5 & 1 & 2 & 4\end{array}\right\}$ significa que en la Malla 1 el interruptor a abrir es aquel que su identificación está en la posición número 3; en la Malla 2 será aquel interruptor cuya identificación está en la posición número 5; y así sucesivamente.

De las mallas así definidas se encuentra que su longitud (el número de ramas que las componen) no es igual para cada una de ellas. Esta longitud determinará el límite superior de cada variable (gen) que será dato de entrada para la utilización del AG, (el límite inferior es 0).

A partir de la cadena que arroja el algoritmo, se determinan los interruptores a abrir y se la somete al cálculo del flujo de potencia. El objetivo entonces, consiste en obtener una configuración tal que la reducción del valor de pérdidas esté entre un 6\% y 15\%, en relación con la configuración inicial.

\section{Parámetros de simulación óptimos}

Definidos los parámetros inherentes a la topología y codificación de las variables (longitud de la cadena, límites superior e inferior de cada variable y flujo de potencia), se procede a determinar los propios del AG: población, número de generaciones, tipo de variable de entrada, tipos y rangos de selección, de cruzamiento y de mutación. A los efectos de encontrar aquellos que otorgan la mejor performance al desarrollo del algoritmo, se analizó el comportamiento de los parámetros mencionados.

Para la correcta elección de método de selección (Ruleta, Torneo, Estocástica Uniforme, Uniforme, Remaider) se realizaron varias corridas para cada opción y se tomaron los tiempos en alcanzar el óptimo. Los resultados se observan en la Tabla 1. Del análisis de la misma surge que la selección Uniforme queda descartada por tener una probabilidad del 10\% de encontrar la solución óptima sobre el total de las corridas realizadas.

Se encontró que la elección del Método de Selección no influye en demasía sobre el tiempo total de cálculo ya que difieren en promedio, uno de otro, en un valor despreciable. Por tanto, para el sistema 33-B se utilizó la selección por Torneo, debido a que las variables son escasas y por ende el espacio de búsqueda es reducido. Además el tiempo obtenido puede reducirse aún más modificando la cantidad de individuos que participan en el torneo.

Para la elección más adecuada del tipo de cruzamiento, se realizaron varias corridas (Tabla 2), encontrándose que tanto el tipo diseminado, un punto y dos puntos de corte presentan en tiempos una mejor performance, pero no muestran una mayor eficiencia uno frente al otro.

Para este trabajo se optó por utilizar un cruzamiento en dos puntos, dado que en el caso de no encontrar la solución óptima, la más cercana a ésta lo encontrará en 10,625 seg. frente a los 20,015 seg. del tipo diseminado.

En cuanto a la fracción de cruzamiento, se partió de individuos que no se reproducen para generar nuevas poblaciones ( $0 \%$ de la población) hasta nuevos conjuntos generados con la totalidad de sus integrantes (100\% de la población). Es decir de 0 a 1, a pasos de 0,05. 
Tabla 1: Tiempos en alcanzar el óptimo [seg.]. Tipo Selección

(Los valores indicados con asterisco “*” pertenecen a configuraciones distintas de la óptima)

\begin{tabular}{|c|c|c|c|c|c|}
\hline & \multicolumn{5}{|c|}{ Tipos de Selección } \\
\hline Nro. corrida & Ruleta & Torneo & $\begin{array}{c}\text { Estocástica } \\
\text { uniforme }\end{array}$ & Remainder & Uniforme \\
\hline 1 & 10.407 & 13.156 & 9.797 & 18.078 & *14.078 \\
\hline 2 & 11.078 & 8.172 & 16.516 & 11.047 & 10.703 \\
\hline 3 & 16.422 & 10.016 & $\star 7.547$ & 19.172 & $\star 8.406$ \\
\hline 4 & 17.469 & 10.922 & 9.938 & 15.328 & *10.765 \\
\hline 5 & 11.484 & 8.312 & 10.344 & 16.156 & *11.891 \\
\hline 6 & 8.031 & 7.813 & 16.469 & 12.954 & *13.984 \\
\hline 7 & 11.437 & 14.546 & 8.375 & 10.234 & $\star 12.765$ \\
\hline 8 & 9.187 & *10.625 & 13.797 & 10.687 & *11.875 \\
\hline 9 & 14.735 & 11.406 & 10.969 & 9.531 & *19.515 \\
\hline 10 & 12.281 & 8.719 & 11.156 & 12.235 & *12.656 \\
\hline $\begin{array}{c}\text { Promedio } \\
\text { [seg.] }\end{array}$ & 12.253 & $\begin{array}{r}10.340 \\
\star 10.625 \\
\end{array}$ & $\begin{array}{r}11.929 \\
* 7.547 \\
\end{array}$ & 13.542 & $\begin{array}{r}10.703 \\
\star 12.881 \\
\end{array}$ \\
\hline
\end{tabular}

Tabla 2: Tiempos en alcanzar el óptimo [seg.]. Tipo Crossover

(Los valores indicados con asterisco "*” pertenecen a configuraciones distintas de la óptima)

\begin{tabular}{|c|c|c|c|c|c|}
\cline { 2 - 6 } \multicolumn{1}{c|}{} & \multicolumn{5}{c|}{ Tipos de Crossover } \\
\hline Nro. Corrida & Diseminada & Un punto & Dos puntos & Intermedia & Heurística \\
\hline 1 & $\star 20.015$ & 8.672 & 13.156 & 9.302 & ${ }^{*} 9.891$ \\
\hline 2 & 9.672 & $\star 11.422$ & 8.172 & ${ }^{\star} 9.176$ & 9.609 \\
\hline 3 & 9.406 & $\star 7.906$ & 10.016 & 12.156 & 18.340 \\
\hline 4 & 15.719 & 8.188 & 10.922 & 10.671 & 12.336 \\
\hline 5 & 8.547 & 9.422 & 8.312 & ${ }^{*} 9.141$ & 13.708 \\
\hline 6 & 11.078 & 14.532 & 7.813 & ${ }^{*} 9.883$ & 15.746 \\
\hline 7 & 14.281 & 8.578 & 14.546 & ${ }^{\star 9} .401$ & 10.151 \\
\hline 8 & 9.109 & 9.500 & $\star 10.625$ & ${ }^{\star} 10.226$ & 11.352 \\
\hline 9 & 9.969 & 9.531 & 11.406 & 12.259 & 10.433 \\
\hline 10 & 9.422 & 7.812 & 8.719 & ${ }^{\star} 8.346$ & $\star 10.123$ \\
\hline Promedio & 10.800 & 9.529 & 10.340 & 11.097 & 12.709 \\
[seg.] & $\star 20.015$ & $\star 9.664$ & $\star 10.625$ & $\star 9.362$ & $\star 10.007$ \\
\hline
\end{tabular}

Los resultados son volcados en la Figura 2, en la que se encuentran representados tres valores de pérdidas: a) para la solución óptima que es de $134,7321 \mathrm{~kW}$; b) el valor siguiente de pérdidas para la configuración de apertura de interruptores más cercana a la solución óptima, que es de 134,9930 $\mathrm{kW}$; c) el valor de una configuración que no se encuentra cerca de la óptima y posiblemente sea un óptimo local, 137,5293 kW. Las ordenadas representan el número de veces que es logrado ese valor de pérdidas en las 5 corridas y las abscisas representan la fracción de cruzamiento.

De la gráfica puede deducirse que para fracciones de cruzamiento menores a 0,7 se encuentra (para las 5 corridas) siempre el óptimo, y para valores superiores a 0,9 , el número de veces que es alcanzada la solución óptima decae, a la vez que aparecen soluciones próximas a la óptima.

Cabe aclarar que hasta una fracción de cruzamiento menor o igual a 0,7, el tiempo computacional involucrado para obtener dicha solución es muy importante, mientras que para valores mayores a 0,7 
el tiempo que le lleva al AG obtener una solución óptima, o muy cercana a la óptima, es significativamente menor. Es más, para el valor de fracción de cruzamiento igual a 1, la solución es hallada muy rápidamente, pero en ninguna de las corridas se alcanza la solución óptima.

También puede observarse que en el rango determinado por los valores entre 0,8 y 0,95, se encuentra la solución óptima con un tiempo de búsqueda aceptable.

De este análisis se concluye que debe existir un compromiso en la elección de la fracción de cruzamiento adecuada, tanto para obtener una solución muy buena como para disminuir el tiempo computacional a un valor aceptable. Por lo tanto, del intervalo encontrado $[0,7 ; 0,9]$ a adoptarse para el funcionamiento del AG, se tomó un valor de fracción de cruzamiento igual a 0,85.

También se tuvo en cuenta el tipo de Cruzamiento (crossover), donde nuevamente se realizaron varias corridas variando su tipo (diseminado, un punto, dos puntos, intermedio, heurístico). Se determinó los tiempos empleados para lograr el óptimo (Tabla 2).

Del análisis realizado surge que la mejor performance de tiempos la demuestran el tipo diseminado, un punto y dos puntos. De los tres, no hay un método que se destaque sobre los demás con respecto al ahorro de tiempo de cálculo, a pesar de que el método por un punto es el que, en promedio, presenta más ventaja y muestra un $90 \%$ de probabilidad de encontrar el resultado óptimo.

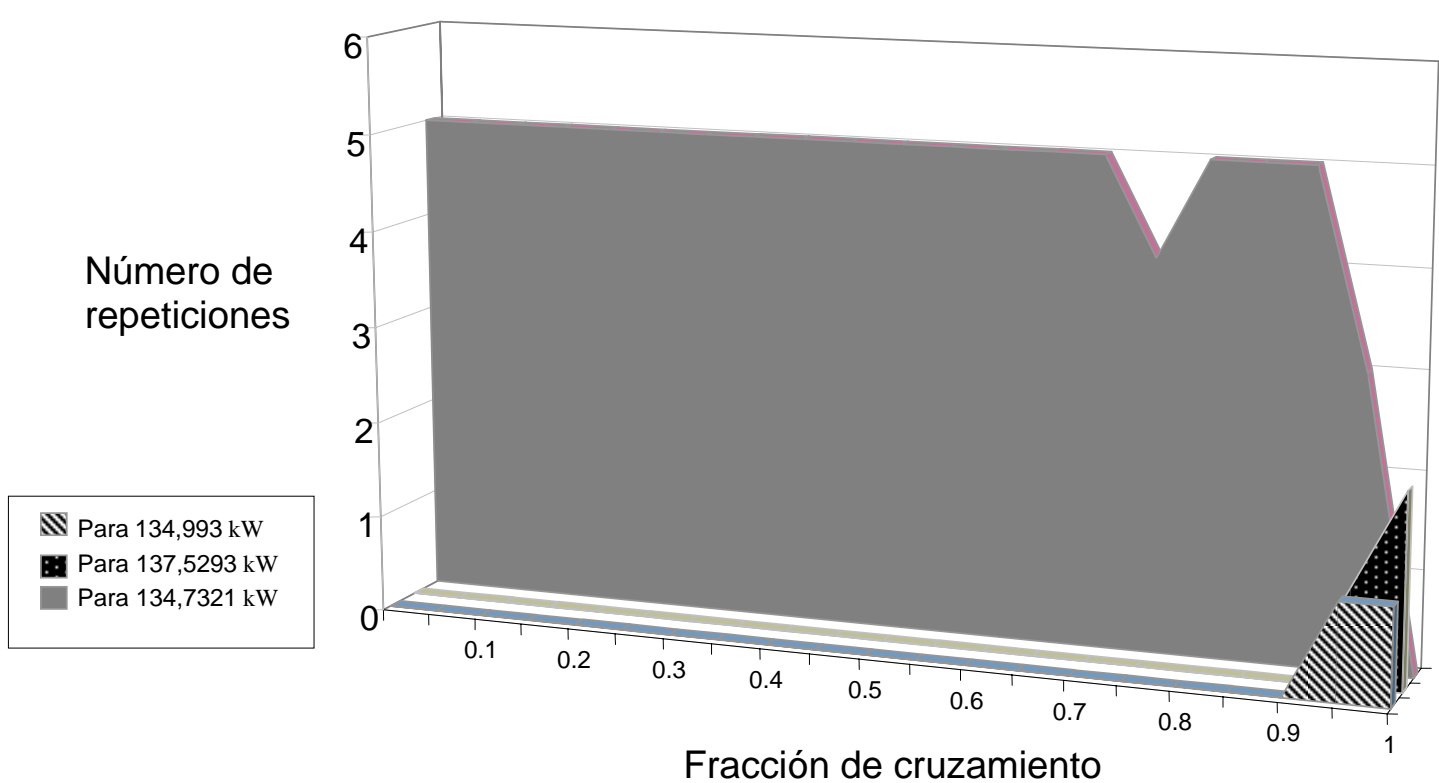

Fig. 2: Rendimiento del AG mediante la variación de la fracción de cruzamiento.

Ambas opciones (diseminado y dos puntos) traerán aparejados los mismos resultados, pero para los fines de este trabajo se utilizará el tipo dos puntos, ya que en el caso de no encontrarse la solución óptima, la más cercana a ésta lo será en la mitad de tiempo $(10,625$ seg.) que si se opta por el método diseminado (20,015 seg.).

En cuanto al Tipo y Rango de Mutación las opciones disponibles son dos: Uniforme y Gaussiana. En este caso, la elección recayó en la Uniforme, ya que la Gaussiana disminuye la tasa de mutación a medida que avanzan las generaciones, por lo que algunas regiones del espacio de búsqueda dejan de ser exploradas, eliminando soluciones potenciales para resolver el problema. Por otro lado, la Uniforme mantiene constante la mutación.

Con respecto a la Tasa de Mutación cabe destacar que ésta debe ser lo bastante alta para promover una cantidad suficiente de diversidad en la población sin acabar con los individuos; según De Jong 
(1975) debe ser $l^{-1}$, donde I es la longitud de la cadena, resultando ser 0,2 para el sistema 33-B.

\section{ANÁLISIS Y DISCUSIÓN}

La configuración obtenida de la primera corrida del algoritmo indicó los siguientes interruptores a abrir: 7, 9, 14, 32 y 37 (Figura 3), resultando ser la óptima. Por tanto, no existe otra configuración que pueda disminuir el valor de las pérdidas de potencia por debajo de los 134,7321 kW.

Por otro lado, se analizaron los perfiles de tensiones, encontrándose que el valor más bajo fue de 0,9424 p.u. (barra 31), lo que implica una caída de tensión en la barra de 6\%, el cual es menor al valor máximo permitido por la normativa nacional: $8 \%$ en media tensión. En otros países, este valor disminuye al 5\%. Una nueva corrida se hizo teniendo en cuenta esta restricción, encontrándose que no existe una configuración que tenga alguna barra con un nivel de caída de tensión menor o igual a este valor. La configuración que más se acerca al cumplimiento de esta restricción corresponde a la apertura de los interruptores 7, 9, 14, 28 y 32 con un valor de pérdidas de 134,9930 kW, ya que la barra con menor tensión acusa una caída de tensión de 5.76\% (barra 31).

Análogamente, se realizó el mismo análisis para el sistema de 69-Bus, arrojando el resultado que se muestra en la Tabla 3. En este caso, la topología de la red determinada por la apertura de los interruptores 58-61-72-14-74, satisface la condición de configuración óptima y a su vez con las de caída de tensión $(-\Delta \mathrm{V})$ máxima del $5 \%$ y $8 \%$ impuestas como restricciones. La hipótesis sobre disminución de pérdidas ha sido satisfecha de manera favorable ya que el $51.3 \%$ alcanzado supera el rango del $6 \%$ al $15 \%$ propuesto como objetivo.

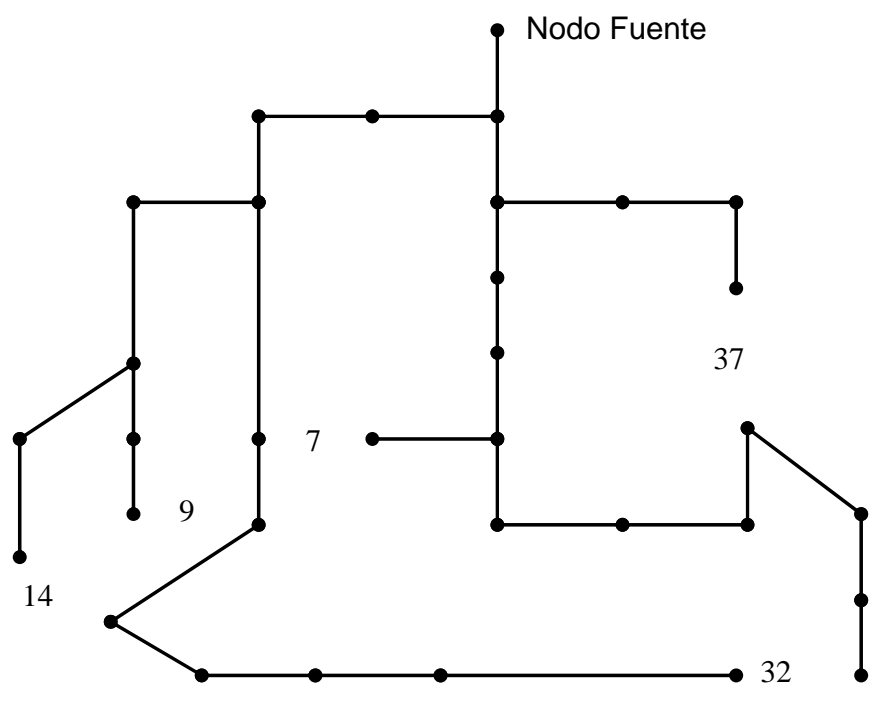

Fig. 3. Interruptores a abrir.

Tabla 3: Configuración Resultante para 69-Bus

\begin{tabular}{|l|c|c|c|}
\hline \multicolumn{2}{|l|}{ Configuraciones } & Pérdidas [kW] & $\begin{array}{c}\text { Reducción } \\
\text { Pérdidas \% }\end{array}$ \\
\hline Inicial & $70-71-72-73-74$ & 188,2002 & 51,3 \\
\hline Óptima & $58-61-72-14-74$ & 91,6489 & 51,3 \\
\hline Restricción $-\Delta \mathrm{V}=8 \%$ & $58-61-72-14-74$ & 91,6489 & 51,3 \\
\hline Restricción $-\Delta \mathrm{V}=5 \%$ & $58-61-72-14-74$ & 91,6489 & \\
\hline
\end{tabular}




\section{CONCLUSIONES}

La aplicación desarrollada mediante AG optimiza los SD tanto en lo que respecta a pérdidas de potencia como al perfil de tensiones. Los resultados obtenidos demuestran que su aplicación en SD eléctricos primarios puede hacerse de manera fiable y su aplicación en tiempo real es posible debido a que los tiempos necesarios para obtener las configuraciones óptimas son aceptables.

La opción de utilizar una simplificación del FdP trae aparejadas ventajas y desventajas. Como principal ventaja, se cita la disminución del tiempo de cálculo ya que las expresiones matemáticas son más simples. En contrapartida, se pierde exactitud y posiblemente las opciones de apertura final de interruptores difieran de las proporcionadas al utilizar un método exacto. En consecuencia, se tienen que tomar los recaudos necesarios si se requiere utilizar un FdP simplificado.

Analizando particularmente los casos bajo estudio en este trabajo para el sistema 33-B, la reducción de pérdidas respecto de la configuración inicial es de un 29,34 \% y para el sistema 69-B, de un $51,30 \%$. Estos porcentajes evidencian que el AG tiene un muy buen comportamiento, con tiempos de cálculo aceptables: 15,812 seg. y 25,781 seg.

Los métodos de selección, cruzamiento, mutación, y sus correspondientes tasas, fueron justificados debidamente mediante el análisis del comportamiento de los resultados frente a sus variaciones. Para cada SD, la aplicación desarrollada entrega el valor de las pérdidas finales y la cadena indica qué interruptores deben ser abiertos. Adicionalmente, a modo de visualización didáctica, se le han agregado funciones para graficar la red resultante y el perfil de tensiones. Estas gráficas sirven para analizar e identificar los cambios efectuados al reconfigurar el SD.

En los antecedentes analizados, en los que se obtiene una configuración similar, no se han tenido en cuenta las restricciones de caída de tensión propuesta en este trabajo.

Debido a la flexibilidad de los AG, es posible aplicar soluciones en las cuales se priorice el servicio a algunas cargas críticas en el caso de cortes de servicio. Esta temática está prevista investigar en trabajos futuros.

\section{NOMENCLATURA}

$\begin{array}{ll}P_{i} \text { y } Q_{i}: & \text { Potencia activa y reactiva de la rama. } \\ P_{L i} \text { y } Q_{L i} & \text { Potencia activa y reactiva de carga } \\ V_{i} & \text { Tensión en la rama. } \\ r_{i} \text { y } x_{i} & \text { Resistencia y reactancia de la rama. }\end{array}$

\section{REFERENCIAS}

Ah King R.T.; B. Radha y H.C.S. Rughooputh; A Real-Parameter Genetic Algorithm for Optimal network reconfiguration, IEEE Conf. on Industrial Tech. ICIT'03, 54-59, Maribor, Slovenia (2003).

Baldwin T.L. y S.A. Lewis; Distribution Load Flow Methods for Shipboard Power Systems. IEEE Trans. on Industry Applications: 40 (2), 1183-1189 (2004).

Baran, M.E. y F.F. Wu; Network, Reconfiguration in Distribution Systems for Loss Reduction and Load Balancing, IEEE Trans. On Power Delivery: 4 (2), 1401-1407 (1989).

Enacheanu, B. y otros cinco autores; Radial Network Reconfiguration Using Genetic Algorithm Based on the Matroid Theory, IEEE Trans. On Power System: 23 (1), 186-195 (2008).

Chiang H.D. y R.J. Jumeau; Optimal Network Reconfi. in distribution Systems: Part 1: A New Formulation and A Solution Methodology, IEEE Trans. On Power Delivery: 5 (4), 1902-1909 (1990a). 
Chiang H.D. y R.J. Jumeau; Optimal Network Reconf. in distribution Systems: Part 2: Solution Algorithms and Numerical Results, IEEE Trans. On Power Delivery: 5 (3), 1568-1574 (1990b).

De Jong, K. A., An Analysis Of The Behavior of a Class of Genetic Adaptive Systems, Doctoral dissertation, Dep. of Computer and Communic. Sciences, University of Michigan, Ann Arbor. (1975).

Goswami, S.K. y S.K. Basu; A New Algorithm for the Reconfiguration of Distribution Feeders for Loss Minimization, IEEE Trans. On Power Delivery: 7 (3), 1482-1491 (1992).

Hong Y.Y. y S.Y. HO; Genetic Algorithm Based Network Reconfiguration for Loss Minimization in Distrib. Systems, Power Eng. Society Gral. Meeting 2003, IEEE, 1,486-490, 13 al 17 de Julio (2003).

Hong Y.Y. y S.Y. HO; Determination of Network Configuration Considering Multiobjective in Distribution System Using Genetic Algorithms, Trans. on Power System: 20 (2), 1062-1069 (2005).

Hsiao Y.T. y C.Y. Chen; Multiobjective Optimal Feeder Reconfiguration, IEE Proceedings-Generation, Transmission and Distribution: 148 (4), 333-336 (2001).

Jeon Y.J. y J.C. Kim; Network Reconfiguration in Radial Distribution System Using Simulated Annealing and Tabu Search, Actas del Power Engineering Society Winter Meeting, 2000 IEEE, 4, 2329-2333, 23 al 27 de Enero (2000).

Kashem M.A.; V. Ganapathy y G.B. Jasmon; Network Reconfiguration for Load balancing in Distribution Networks, IEE Proceedings-Generation, Transm. and Distrib.: 146 (6), 563-567 (1999).

Kashem M.A.; V. Ganapathy y G.B. Jasmon; A Novel Method for loss Minimization in Distribution Networks, International Conference on Electric Utility Deregulation and Restructuring and Power Technologies 2000, 251-256, London, Inglaterra, 4 al 7 Abril (2000).

Li L.; G. Zhizhong y W. Chengmin; A New Topology Structure and Load Flow Solution for Radial Distribution Networks, Power System Technology: 2, 1064-1067 (2002).

Lin W.M., F.S. Cheng y M.T. Tsay; Distribution Feeder Reconfiguration with Refined Genetic Algorithm, IEE Proceedings-Generation, Transmission and Distribution: 147 (6), 349-354 (2000).

Morton, A. B. y I.M.Y. Mareels; An Efficient Brute-Force Solution To The Network Reconfiguration Problem, IEEE Trans. On Power Delivery: 15 (3), 996-1000 (2000).

Radha B., R.T.F. Ah King y H.C.S Rughooputh; A Modified Genetic Algorithm for Optimal Electrical Distribution Network Reconfiguration, Actas del IEEE Evolutionary Computation CEC '03, 2, 14721479, 8 al 12 de Diciembre (2003).

Taylor T. y D. Lubkeman; Implementation of Heuristic Search Strategies for Distribution Feeder Reconfiguration. IEEE Trans. On Power Delivery: 5 (1), 239-245 (1990). 